\title{
SS, Rat Strain
}

National Cancer Institute

\section{Source}

National Cancer Institute. SS, Rat Strain. NCI Thesaurus. Code C76186.

Derived by Rapp from a colony of Sprague-Dawley rats that were initially derived by LK Dahl at Brookhaven National Laboratories. The SS rat strain has been selected for its acute salt sensitivity, leading to the development of hypertension, diabetes, nephropathy, cardiac hypertrophy, heart failure, cholesterolemia, hyperinsulinemia, hypertriglyceridemia. This rat strain is used as an animal model in the study of human hypertension. 